\title{
Toward to Utilize the Heterogeneous Multiple Processors of the Chip Multiprocessor Architecture
}

\author{
Slo-Li Chu \\ Department of Information and Computer Engineering, \\ Chung Yuan Christian University, Chung-Li, Taiwan, R.O.C. \\ slchulcycu.edu.tw
}

\begin{abstract}
Continuous improvements in semiconductor fabrication density are supporting new classes of Chip Multiprocessor (CMP) architectures that combine extensive processing logic/processor with high-density memory in a single chip. One of the architecture, called Processor-in-Memory (PIM) can support high-performance computing by combining various processors in a single system. Therefore, a new strategy is developed to identify their capabilities and dispatch the most appropriate jobs to them in order to exploit them fully. This paper presents a novel scheduling mechanism, called Swing Scheduling to fully utilize all of the heterogeneous processors in the PIM architecture. Integrated with our Octans system, this mechanism can decompose the original program into blocks and can produce a feasible execution schedule for the host and memory processors, even for other CMP architectures. The experimental results for real benchmarks are also proposed.
\end{abstract}

Keywords: Chip Multiprocessor (CMP), Processor-in-Memory, Swing Scheduling, Octans.

\section{Introduction}

In current high-performance computer architectures, the processors run many times faster than the computer's main memory. This performance gap is often referred to as the Memory Wall [25]. This gap can be reduced using the System-on-a-Chip or Chip Multiprocessor [13] strategies, which integrates the processors and memory on a single chip. The rapid growth in silicon fabrication density has made this strategy possible. Accordingly, many researchers have addressed integrating computing logic/processing units and high density DRAM on a single die [5][7][8][9] [10][12][13]. Such architectures are also called Processor-in-Memory (PIM), or Intelligent RAM (IRAM).

Integrating DRAM and computing logic on a single integrated circuit (IC) die generates PIM architecture with several desirable characteristics. First, the physical size and weight of the overall design can be reduced. As more functions are integrated on each chip, fewer chips are required for a complete design. Second, very wide onchip buses between the CPU and memory can be used, since DRAM is located with computing logic on a single die. Third, eliminating off-chip drivers reduces the power consumption and latency [12]. 
This class of architectures constitutes a hierarchical hybrid multiprocessor environment by the host (main) processor and the memory processors. The host processor is more powerful but has a deep cache hierarchy and higher latency when accessing memory. In contrast, memory processors are normally less powerful but have a lower latency in memory access. The main problems addressed here concern the method for dispatching suitable tasks to these different processors according to their characteristics to reduce execution times, and the method for partitioning the original program to execute simultaneously on these heterogeneous processor combinations.

Previous studies of programming for PIM architectures [4][6] have concentrated on spawning as many processors as possible to increase speedup, rather than on the capability difference between the host and memory processors. However, such an approach does not exploit the real advantages of PIM architectures. This study integrates our Octans system that integrates statement splitting, weight evaluation and a scheduling mechanism. The original scheduling [2] mechanism is improved to generate a superior execution schedule to fully utilize all heterogeneous processors in the PIM architecture, using our new Swing Scheduling mechanism. A weight evaluation mechanism is established to obtain a more precise estimate of execution time, called weight. The Octans system can automatically analyze the source program, generate a Weighted Partition Dependence Graph (WPG), determine the weight of each block, and then dispatch the most suitable blocks for execution on the host and memory processors.

The rest of this paper is organized as follows: Section 2 introduces PIM architectures. Section 3 describes our Octans system and the Swing Scheduling algorithms. Section 4 presents experimental results. Conclusions are finally drawn in Section 5 .

\section{The Processor-in-Memory Architecture}

Fig. 1 depicts the organization of the PIM architecture evaluated in this study. It contains an off-the-shelf processor, P.Host, and four PIM chips. The PIM chip integrates one memory processor, P.Mem, with 64 Mbytes of DRAM. The techniques presented in this paper is suitable for the configuration of one P.Host and multiple P.Mems, and can be extended to support multiple P.Hosts.

Table 1 lists the main architectural parameters of the PIM architecture. P.Host is a six-issue superscalar processor that allows out-of-order execution and runs at $800 \mathrm{MHz}$, while P.Mem is a two-issue superscalar processor with in-order capability and runs at $400 \mathrm{MHz}$. There is a two-level cache in P.Host and a one-level cache in P.Mem. P.Mem has lower memory access latency than P.Host since the former is integrated with DRAM. Thus, computation-bound codes are more suitable for running on the P.Host, while memory-bound codes are preferably running on the P.Mem to increase efficiency.

The PIM chip is designed to replace regular DRAMs in current computer systems, and must therefore conform to a memory standard that involves additional power and ground signals to support on-chip processing. One such standard is Rambus [5], so the 


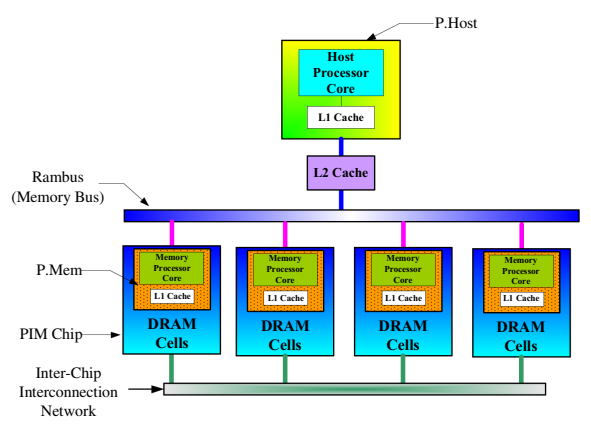

Fig. 1. Organization of the PIM architecture
Table 1. Parameters of the PIM architecture

\begin{tabular}{|c|c|c|}
\hline P.Host & P.Mem & Bus \& Memory \\
\hline |Working Freq: & Working Freq: & Bus Freq: \\
\hline $800 \mathrm{MHz}$ & $400 \mathrm{MHz}$ & $100 \mathrm{MHz}$ \\
\hline Dynamic issue & & P.Host Mem RT: \\
\hline ||Width: 6 & Static issue Width: 2 & $\begin{array}{l}\text { 262. } 5 \mathrm{~ns} \\
\text { P.Mem Mem RT: }\end{array}$ \\
\hline Integer unit num: 6 & Integer unit num: 2 & $50.5 \mathrm{~ns}$ \\
\hline |Floating unit num: 4 & Floating unit num: 2 & $\begin{array}{l}\text { Bus Width: } 16 \text { B } \\
\text { Mem_Data_Transfer: }\end{array}$ \\
\hline |FLC_Type: WT & FLC_Type: WT & \\
\hline |FLC_Size: 32 KB & FLC_Size: $16 \mathrm{~KB}$ & $\begin{array}{l}\text { Mem_Row_Width: } \\
4 \mathrm{~K}\end{array}$ \\
\hline |FLC_Line: 64 B & FLC_Line: 32 B & \\
\hline SLC_Type: WB & SLC: N/A & \\
\hline $\begin{array}{l}\text { SLC_SIZe: } 256 \text { KB } \\
\text { SLC_Line: } 64 \text { B }\end{array}$ & & \\
\hline |Replace policy: & & \\
\hline ||LRU & & \\
\hline Branch penalty: 4 & Branch penalty: 2 & \\
\hline \begin{tabular}{|l} 
P.Host_Mem_Delay: \\
88
\end{tabular} & $\begin{array}{l}\text { P.Mem_Mem_Delay } \\
17\end{array}$ & \\
\hline
\end{tabular}

PIM chip is designed with a Rambus-compatible interface. The private interconnection network of the PIM chips is also provided.

\section{The Octans System}

Most current parallelizing compilers focus on the transformation of loops to execute all or some iterations concurrently, in a so-called iteration-based approach. This approach is suited to homogeneous and tightly coupled multi-processor systems. However, it has an obvious disadvantage for heterogeneous multi-processor platforms because iterations have similar behavior but the capabilities of heterogeneous processors are diverse. Therefore, a different approach is adopted here, using the statements in a loop as a basic analysis unit, called statement-based approach, to develop the Octans system.

Octans is an automatic parallelizing compiler, that partitions and schedules an original program to exploit the specialties of the host and the memory processor. At first, the source program is split into blocks of statements according to dependence relations. Then, the Weighted Partition Dependence Graph (WPG) is generated, and the weight of each block is evaluated. Finally, the blocks are dispatched to either the host or the memory processors, according to which processor is more suitable for executing the block. The major difference between Octans and other parallelizing systems is that it uses a statement rather than an iteration as the basic unit of analysis. This approach can fully exploit the characteristics of statements in a program and dispatch the most suitable tasks to the host and the memory processors. Fig. 2 illustrates the organization of the Octans system.

\subsection{Statement Splitting and WPG Construction}

Statement Splitting splits the dependence graph of the given program by the extended node partition mechanism as introduced in [2]. It divides the original program into 


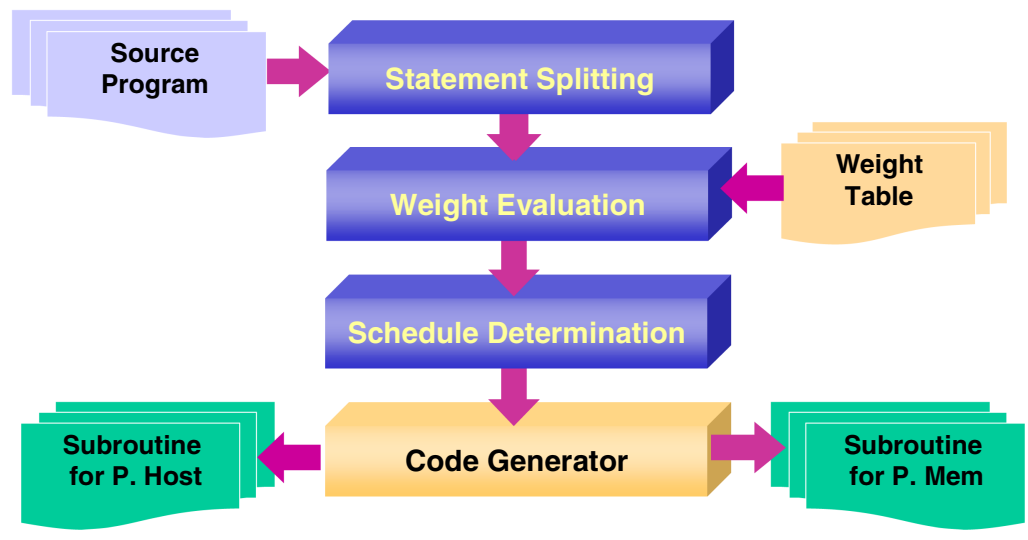

Fig. 2. The sequence of compiling stages in Octans

several small loops within the minimal statements. The detailed mechanisms can be found in literature [2]. Then WPG Construction constructs the Weighted Partition Dependence Graph (WPG), to be used in the subsequent stages of Weight Evaluation, Wavefront Generation and Schedule Determination.

\subsection{Weight Evaluation}

Two approaches to evaluating weight can be taken. One is to predict the execution time of programs by profiling the dominant parts. The other considers the operations in a statement and estimates the program execution time by looking up an operation weight table. The former method called code profiling may be more accurate, but the predicted result cannot be reused; the latter called code analysis can determine statements for suitable processors but the estimated program execution time is not sufficiently accurate. Hence, the Self-Patch Weight Evaluation scheme was designed to combine the benefits of both approaches. It integrates these two approaches together by analyzing code and searching weight table first to estimate the weight of a block. If the block contains unknown operations, the patch (profiling) mechanism is then activated to evaluate the weights of unknown operations. The obtained operation weights are added into the weight table for next look-up. For a detailed description of this scheme, please refer to [2].

\subsection{The Swing Scheduling Mechanism}

Here we propose the Swing Scheduling mechanism to achieve a good schedule for utilizing all of the memory processors in PIM architecture. At first, the redundancy and synchronization between processors are critical factors that affect the performance of job scheduling for multiprocessor platforms. A critical path mechanism is used to minimize the frequency of synchronization. Then the WPG is then partitioned into several Sections according to the nodes on the critical path and the dependence relations between these nodes. In a Section, the blocks are partitioned into several Inner Wavefronts in the following stages. Finally, the execution schedule 
for all P.Host and P.Mems is obtained. If the number of occupied memory processors exceeds the maximum number of processors in the PIM configuration, then the execution schedule will be modified accordingly. Algorithm 1 presents the main steps of this scheduling mechanism.

\section{Algorithm 1. (Swing Scheduling) \\ [Input] \\ $W P G=(P, E)$ : original weighted partition dependence graph after weight is determined. \\ [Output]}

An critical path execution order schedule CPS, where $C P S=\left\{C P S_{1}, C P S_{2}, \ldots, C P S_{i}\right\}$. $C P S_{i}=\left\{C P_{i}, I W F_{i}\right\}$ where $C P_{i}=\left\{\operatorname{Processor}\left(b_{a}\right)\right\}$ where processor is PH or PM .IWF $=\left\{P H\left(b_{a}\right), P M_{l}\left(b_{b}\right), P M_{2}\left(b_{c}\right), \ldots\right\}$ means that in Inner Wavefront $i, P H\left(b_{a}\right)$ means that block $b_{a}$ will be assigned to P.Host, $P M_{l}\left(b_{b}\right)$ means that blocks $b_{b}$ will be assigned to P.Mem $1, P M_{2}\left(b_{c}\right)$ means that blocks $b_{c}$ will be assigned to P.Mem 2 .

\section{[Intermediate]}

W: a working set of nodes ready to be visited.

EO_temp: a working set for execution order scheduling.

iwf_temp: a working set for Inner Wavefront scheduling.

max_EO: the maximum number of execution order.

min_pred_O $\left(b_{i}\right)$ : the minimum execution order for all $b_{i}$ 's predecessor blocks.

max_pred_O $\left(b_{i}\right)$ :the maximum execution order for all $b_{i}$ 's predecessor blocks.

min_succ_RO $\left(b_{i}\right)$ :the minimum execution order for all $b_{i}$ 's successor blocks.

max_succ_RO $\left(b_{i}\right)$ :the maximum execution order for all $b_{i}$ 's successor blocks.

$\operatorname{PHW}\left(b_{i}\right)$ : the weight of $b_{i}$ for P.Host.

$\operatorname{PMW}\left(b_{i}\right)$ : the weight of $b_{i}$ for P.Mem.

$\operatorname{Rank}_{\mathrm{u}}\left(b_{i}\right)$ : the trace up value of $b_{i}$ used for finding CP

$\operatorname{Rank}_{\mathrm{d}}\left(b_{i}\right)$ : the trace down value of $b_{i}$ used for finding CP

\section{[Method]}

Step 1:Call "Initialization()" to initialize the algorithm and determine the weights of each block.

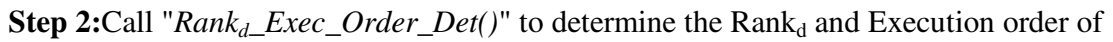
each block.

Step 3:Call "Rank $\_$Det()" to determine the Rank $_{\mathrm{u}}$ of each block.

Step 4:Call "Critical_Path_Det()" to determine the blocks which is belong to the Critical Path.

Step 5:Call "Critical_Path_Block_Sch()" to find out the Section and schedule the Critical Path Block in each Section to the a suitable processor.

Step 6:Call "Inner_Wavefront_Sch()" to partition the blocks which is belong to the same Section into several Inner Wavefront and schedule the blocks in the same Inner Wavefront to the suitable processors.

Step 7:Call "Generate_Schedule()" to generate the execution schedule, CPS.

Step 8:If the occupied processor number is larger than the maximum processor number, call "Modify_schedule()" to modify the original execution schedule to fit the processor number, else Stop the algorithm.

The algorithm includes eight major steps. In Step 1, the algorithm calls "Initialization()" to initiate the necessary variables and determine the P.Host and P.Mem weights of each blocks determined by the weight evaluation mechanism. 
Swing algorithm adopts the critical path method to partition WPG into Sections. Therefore, the critical path and the blocks on the critical path must be determined. Then the attributes, rand $_{u}$ and $r a n k_{d}$, of block $b_{i}$ in WPG are defined by the following equations.

$$
\begin{aligned}
& \operatorname{rank}_{u}\left(b_{i}\right)=P M W\left(b_{i}\right)+\max _{b_{j} \in \text { succ }_{\left(b_{i}\right)}}\left(\operatorname{rank}_{u}\left(b_{j}\right)\right) \\
& \operatorname{rank}_{d}\left(b_{i}\right)=\max _{b_{j} \in \operatorname{pred}_{\left(b_{i}\right)}\left\{\operatorname{rank}_{d}\left(b_{j}\right)+P M W\left(b_{j}\right)\right\}}
\end{aligned}
$$

Here, $\operatorname{succ}\left(b_{i}\right)$ and $\operatorname{pred}\left(b_{i}\right)$ represent all of the successors and predecessors of $b_{i}$, respectively. The critical path is defined as the following equation.

A block $b_{i}$ is on the critical path, if and only if $\operatorname{rank}_{u}\left(b_{i}\right)+\operatorname{rank}_{d}\left(b_{i}\right)=\operatorname{rank}_{u}\left(b_{s}\right)$, where $b_{s}$ is the start block of the WPG, and $b_{i}$ is called the critical path block.

According to the above definitions, the critical path and the critical path block can be determined from Step 2 to Step 4. Step 2 calls "Rank $k_{-}$Exec_Order_Det ()" to determine $\operatorname{rank}_{d}$ and the execution order of each block. Step 3 calls "Rank ${ }_{u \_} \operatorname{Det}()^{\prime \prime}$ to determine $\operatorname{rank}_{u}$ of each block. Then, the algorithm calls "Critical_Path_Det()" to determine which blocks are critical path blocks in Step 4.
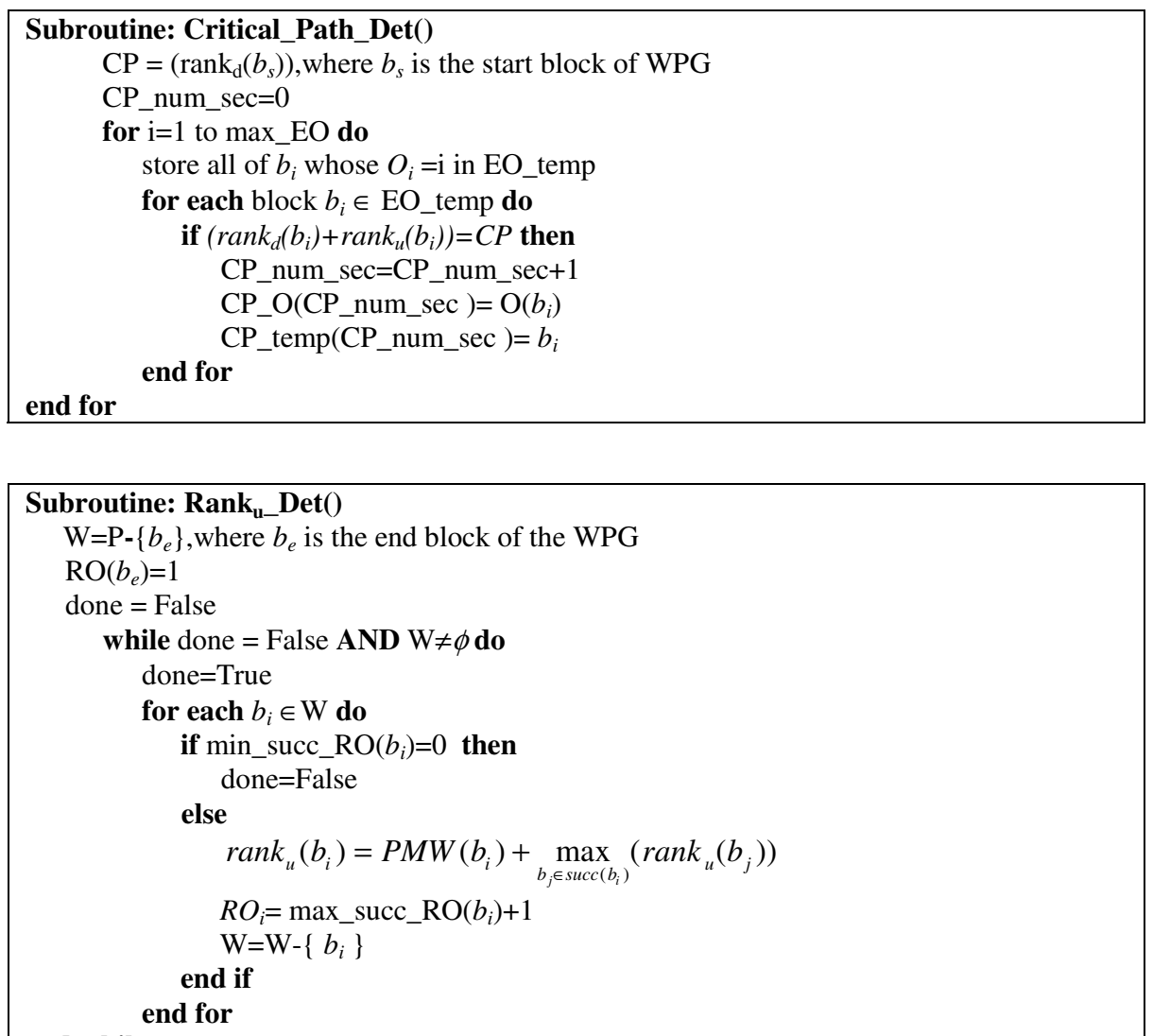


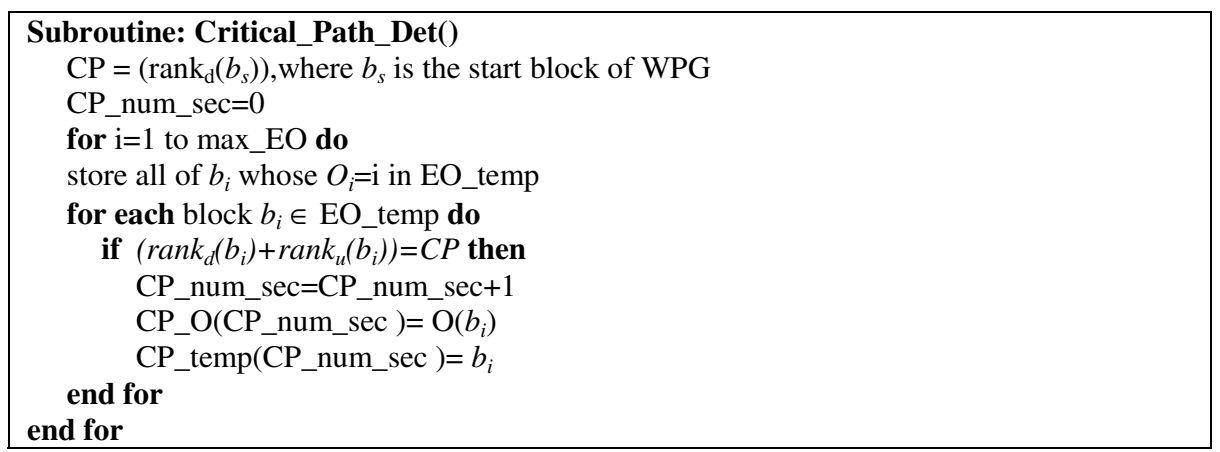

Fig. 3 illustrates the WPG of the synthetic program, which is processing in stages stated above. In this WPG, the shadow blocks are on the critical path. When the critical path is determined in Step 5, "Critical_Path_Block_Sch()" is called to partition all blocks in the WPG into several Sections. Fig. 4 illustrates the result of the given WPG, which is partitioned into five Sections, Section 1:\{b1\}, Section 2: $\{\mathrm{b} 2, \mathrm{~b} 3$, b4, b5, b6, b7, b8, b9, b10, b11, b12, b13, b14\}, Section 3:\{b15\}, Section 4: \{b16, b17, b18, b19, b20, b21, b22, b23, b24, b25, b26, b27, b28 $\}$ and Section 5: $\{$ b29\}. The execution order of Sections is governed by their dependence relations. After the critical path block is identified, the remaining blocks are partitioned into several Inner Wavefronts according to the order of execution and the dependence relations. In Fig. 4, Section 2 of the WPG is used to explain how blocks are scheduled in a Section. Since b2 is the block on critical path in Section 2, "Critical_Path_Block_Sch()" is firstly used to schedule $\mathrm{b} 2$ to reduce the waiting and synchronization frequencies. The remaining blocks are partitioned into three wavefronts according to the $O_{i}$ of each block, by calling "Inner_Wavefront_Sch( $)$ " in Step 6. Finally, iw1 $=\{b 3$, b4, b5, b6 $\}$, iw2 $=\{b 7, b 8, b 9\}$, iw3 $=\{b 10, b 11, b 12, b 13\}$ are determined.

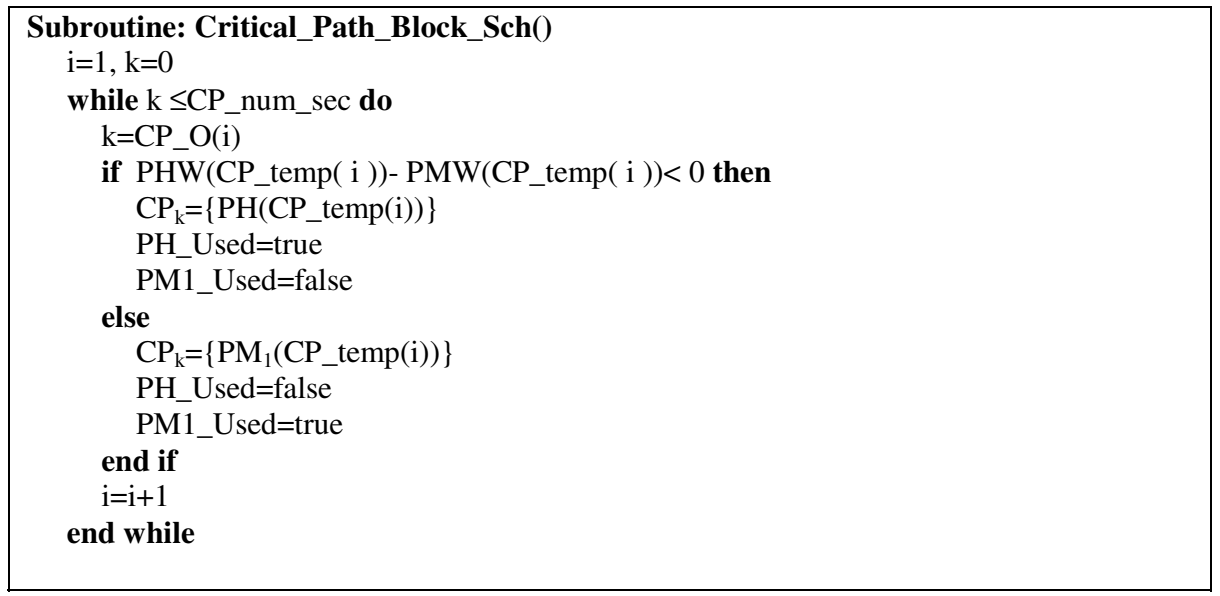




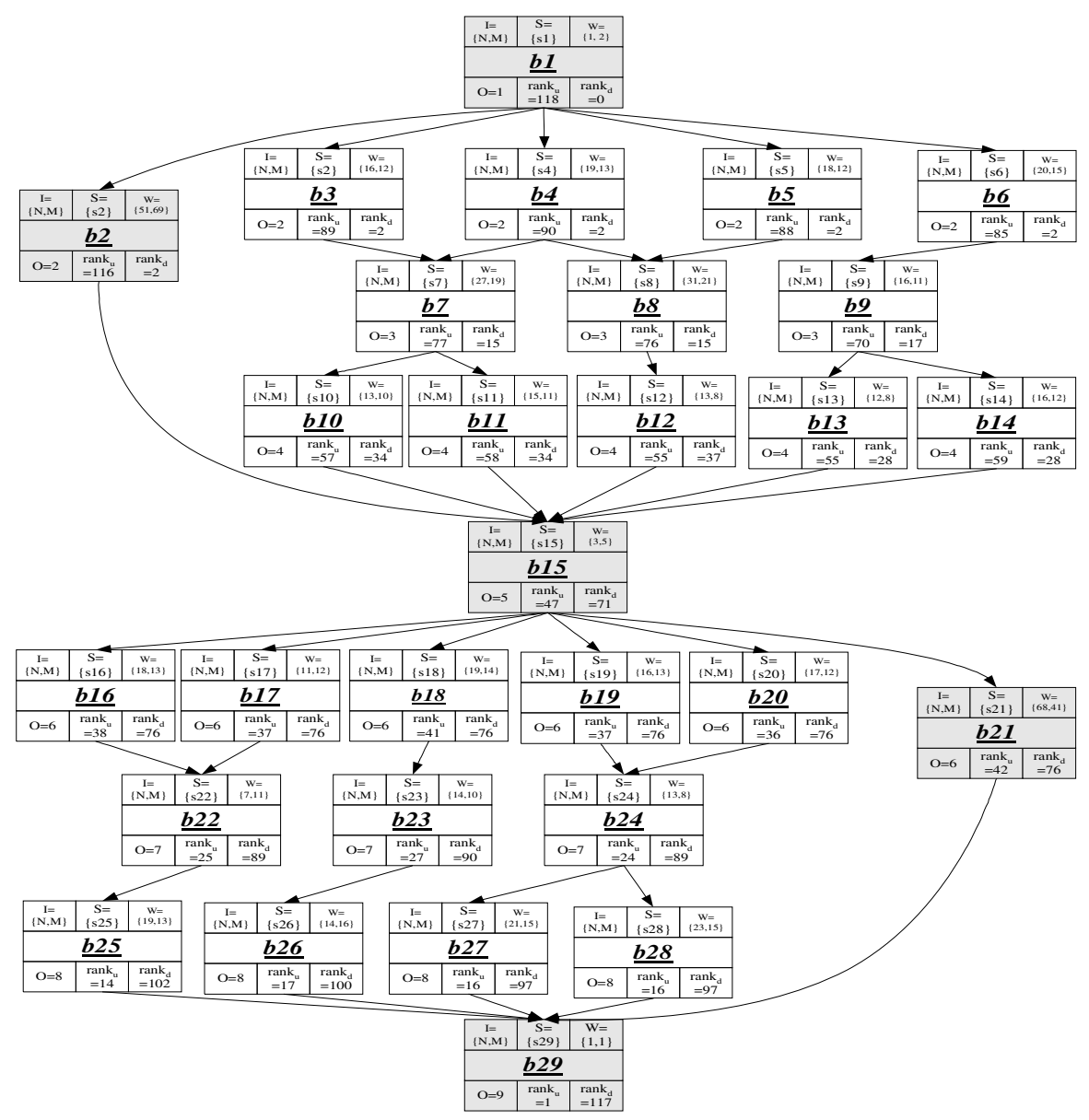

Fig. 3. WPG of a synthetic example

Section 2 $=\{\mathrm{b} 2, \mathrm{~b} 3, \mathrm{~b} 4, \mathrm{~b} 5, \mathrm{~b} 6, \mathrm{~b} 7, \mathrm{~b} 8, \mathrm{~b} 9, \mathrm{~b} 10, \mathrm{~b} 11, \mathrm{~b} 12, \mathrm{~b} 13\}$

Critical path $=\{\mathrm{b} 2\}$

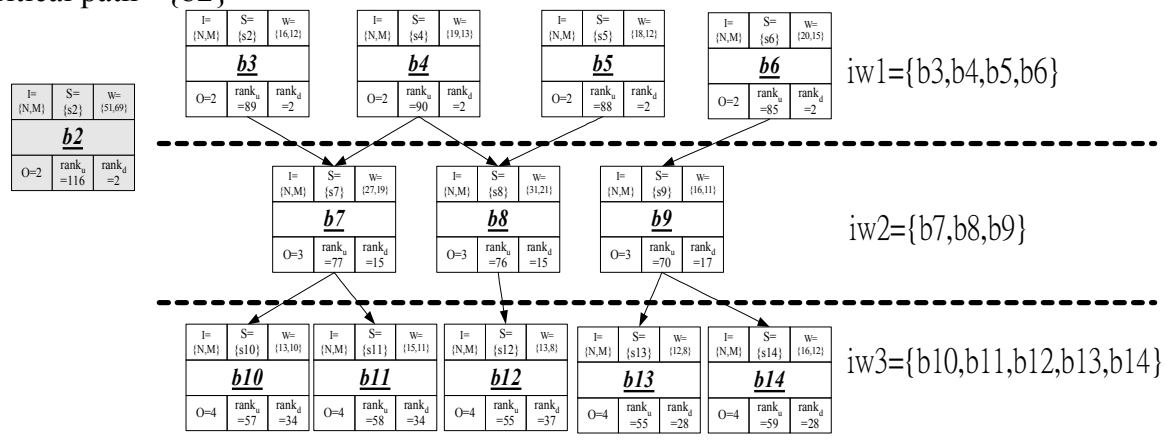

Fig. 4. Scheduled WPG of Section 2 


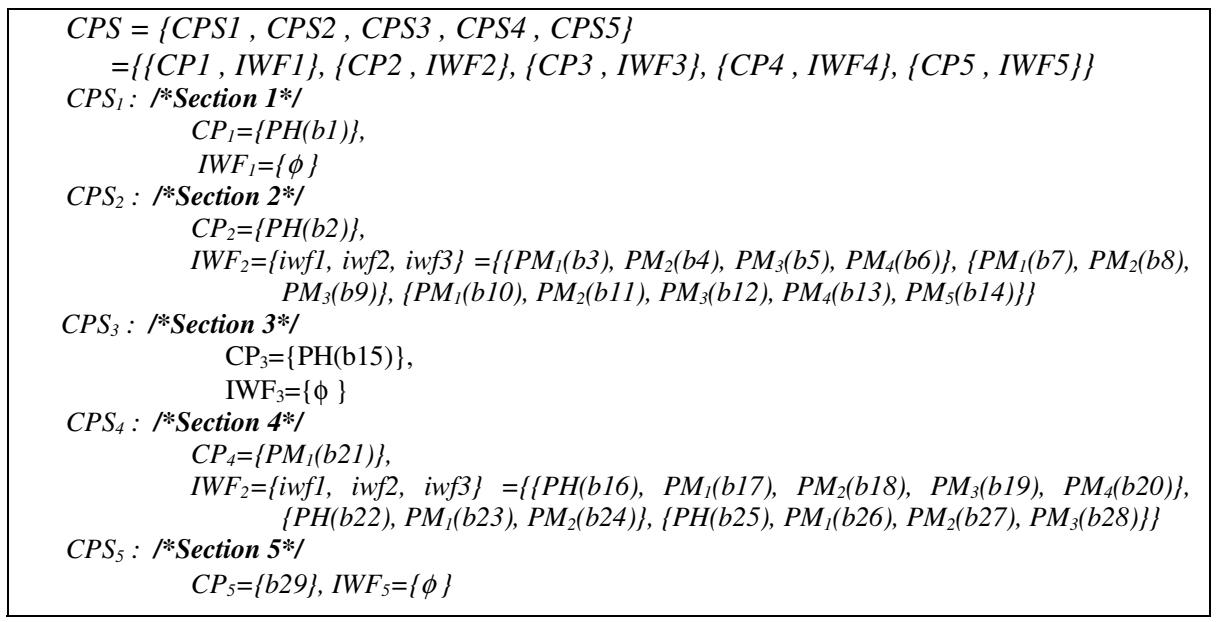

Fig. 5. Output of the Swing scheduling algorithm

In Step 7, the execution schedule is generated by "Generate_Schedule()", as shown in Fig. 5. and Fig. 6 shows the graphical execution schedule. The shaded blocks the Fig. 5 represent the execution latency. The blank blocks indicate that the processor is waiting for other processors to synchronize. The bold and dotted lines determine the point of synchronization of Section and Inner Wavefront respectively.

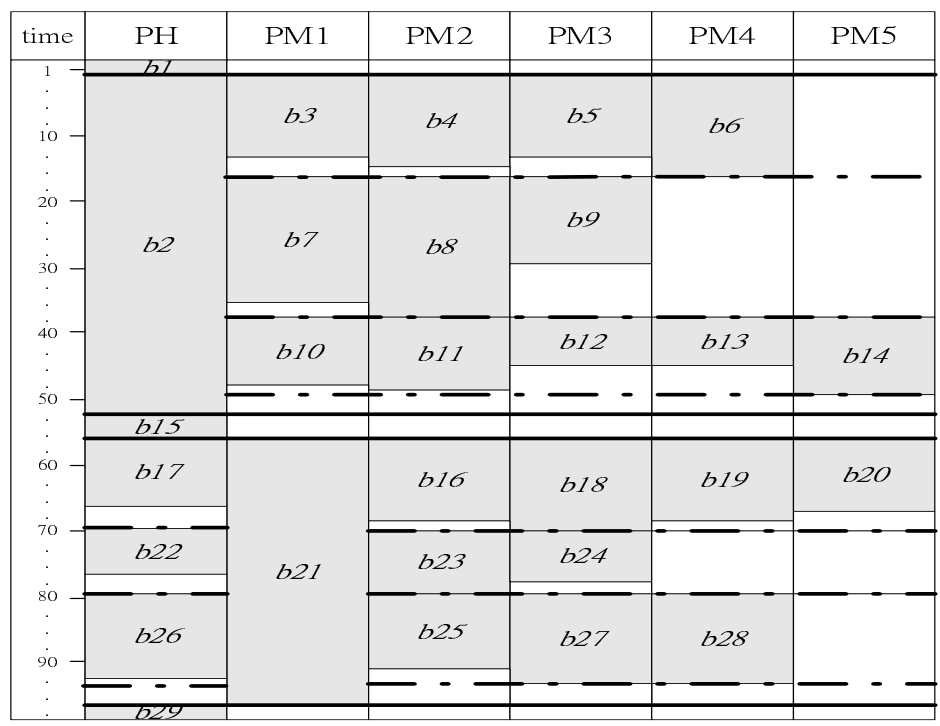

Fig. 6. Graphical execution schedule of the given example 
Sometimes, the execution schedule may occupy more processors than are present in the architectural configuration. Therefore, Step 8 calls "Modify_schedule()" to modify the execution schedule as necessary.

\section{Experimental Results}

The code generated by our Octans system is targeted on our PIM simulator that is derived from the FlexRAM simulator developed by the IA-COMA Lab. at UIUC [13]. Table 1 lists the major architectural parameters. In this experiment, the configuration of one P.Host with many P.Mem processors is modeled to reflect the benefits of the multiple memory processors.

This experiment utilizes multiple P.Mem processors in the PIM architecture to improve performance. The evaluated applications include five benchmarks: $c g$ is from the serial version of NAS; swim is from SPEC95; strsm is from BLAS3; TISI is from Perfect Benchmark, and fft is from [45].

Table 2 and Fig. 7 summarize the experimental results. "Standard" denotes that the application is executed in P.Host alone. This experiment concerns a general situation of a uniprocessor system, and is used to compare speedup. "1-P.Mem" implies that the application is transformed and scheduled by the simplified Swing Scheduling for the one-P.Host and one-P.Mem configuration of the PIM architecture. "n-P.Mem" implies that the application is transformed and scheduled by Swing Scheduling mechanism for the one P.Host and multiple P.Mem configuration of the PIM architecture.

Table 2 and Fig. 7 indicate that swim and $c g$ have quite a good speedup when the Swing Scheduling mechanism is employed because these programs contain many memory references and few dependence relations. Therefore, the parallelism and memory access performance can be improved by using more memory processors. Applying the 1-P.Mem scheduling mechanism can also yield improvements. strsm exhibits an extremely high parallelism but a rather few memory access, so the Swing Scheduling mechanism is more suitably adopted than the 1-P.Mem scheduling mechanism. TISI cannot generate speedup when the 1-P.Mem scheduling mechanism is applied, since it is a typical CPU bounded program, and involves many dependencies. The Swing Scheduling mechanism can be suitably used to increase speedup. Finally, in $f f t$, the program is somewhat computation-intensive and

Table 2. Execution cycles of five benchmarks

\begin{tabular}{|l|r|l|r|r|r|r||}
\hline \multirow{2}{*}{$\begin{array}{l}\text { Bench- } \\
\text { mark }\end{array}$} & \multirow{2}{*}{\begin{tabular}{l} 
Standard \\
\cline { 5 - 7 }
\end{tabular}} & \multirow{2}{*}{$\begin{array}{l}\text { 1-P.Mem } \\
\text { Scheduling }\end{array}$} & $\begin{array}{l}\text { n-P.Mem } \\
\text { Scheduling }\end{array}$ & $\begin{array}{l}\text { 1-P.Mem } \\
\text { Scheduling }\end{array}$ & $\begin{array}{l}\text { n-P.Mem } \\
\text { Scheduling }\end{array}$ & $\begin{array}{l}\text { n(Occupied } \\
\text { P.Mem) }\end{array}$ \\
\hline swim & 228289321 & 116669760 & 52168027 & 1.96 & 4.38 & 6 \\
\hline cg & 91111840 & 51230772 & 32124287 & 1.78 & 2.84 & 4 \\
\hline strsm & 703966766 & 489967053 & 187989176 & 1.44 & 3.74 & 5 \\
\hline TISI & 133644087 & 173503404 & 91098174 & 0.77 & 1.47 & 2 \\
\hline fft & 117998621 & 101841407 & 110399171 & 1.16 & 1.07 & 2 \\
\hline
\end{tabular}



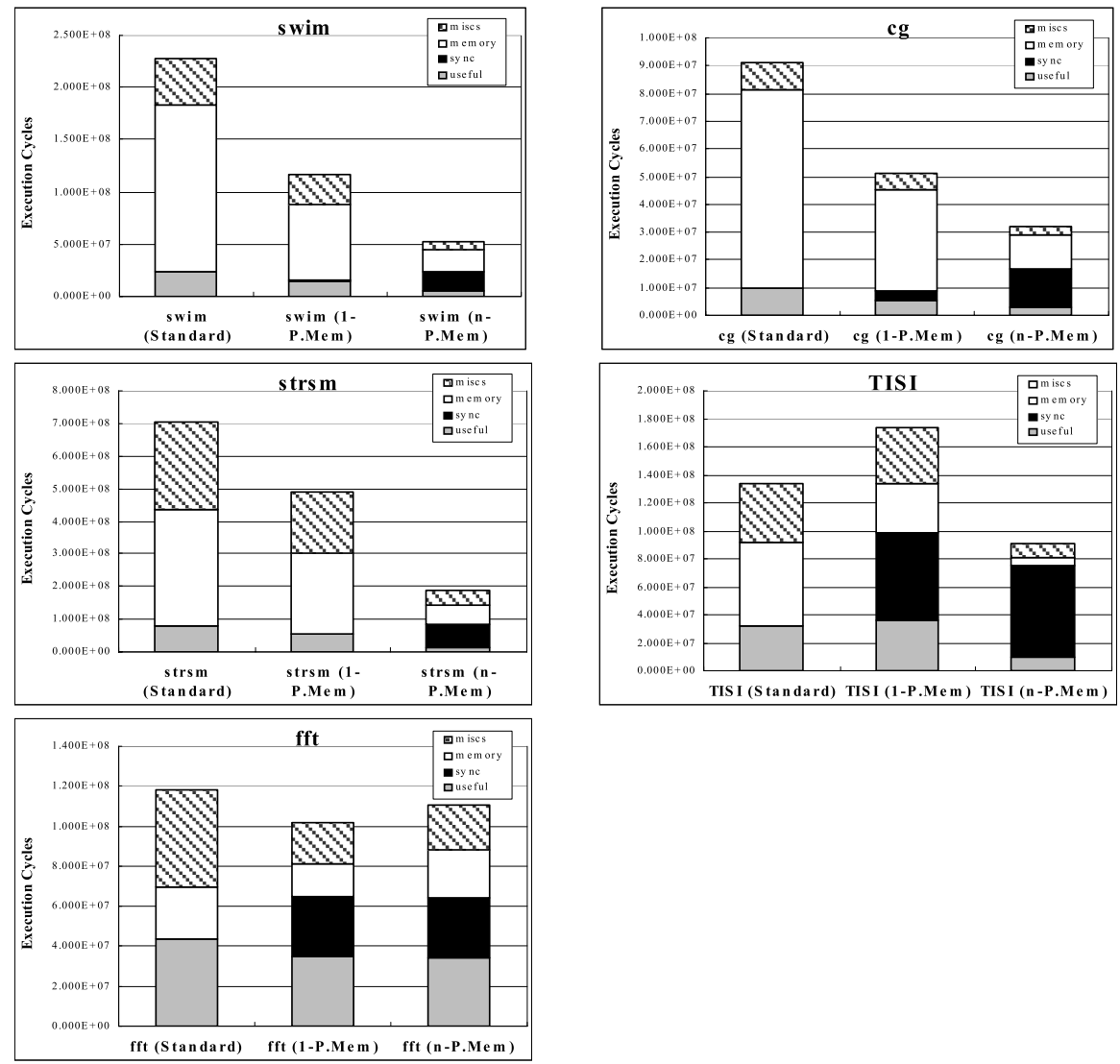

Fig. 7. Execution times of five benchmarks obtained by Standard, 1-P.Mem and n-P.Mem settings.

sequential, and therefore only a little speedup can be improved after the 1-P.Mem scheduling mechanism is applied. However, an additional overhead is generated when the Swing Scheduling mechanism is applied. Accordingly, 1-P.Mem and Swing scheduling mechanisms are suitable for different situations. Choosing the 1-P.Mem or Swing scheduling mechanism more heuristically in the scheduling stage of the Octans system will improve performance.

\section{Conclusions}

This study proposes a new scheduling mechanism, called Swing Scheduling, with Octans system for a new class of high-performance chip multiprocessor architectures, Processor-in-Memory, which consists of a host processor and many memory processors. The Octans system partitions source code into blocks by statement splitting; estimates the weight (execution time) of each block, and then schedules each block to the most suitable processor for execution. Five real benchmarks, swim, TISI, 
strsm, cg, and fft were experimentally considered to evaluate the effects of the Swing Scheduling. In the experiment, the performance was improved by a factor of up to 4.38 while using up to six P.Mems and one P.Host. The authors believe that the techniques proposed here can be extended to run on DIVA, EXECUBE, FlexRAM, and other high-performance chip multiprocessor architectures by slightly modifying the code generator of the Octans system.

\section{Acknowledgements}

This work is supported in part by the National Science Council of Republic of China, Taiwan under Grant NSC 96-2221-E-033 -019-

\section{References}

[1] Blume, W., Eigenmann, R., Faigin, K., Grout, J., Hoeflinger, J., Padua, D., Petersen, P., Pottenger, B., Rauchwerger, L., Tu, P., Weatherford, S.: Effective Automatic Parallelization with Polaris. International Journal of Parallel Programming (May 1995)

[2] Chu, S.L.: PSS: a novel statement scheduling mechanism for a high-performance SoC architecture. In: Proceedings of Tenth International Conference on Parallel and Distributed Systems, pp. 690-697 (July 2004)

[3] Crisp, R.: Direct Rambus Technology: the New Main Memory Standard. In: Proceedings of IEEE Micro, pp. 18-28 (November 1997)

[4] Hall, M., Anderson, J., Amarasinghe, S., Murphy, B., Liao, S., Bugnion, E., Lam, M.: Maximizing Multiprocessor Performance with the SUIF Compiler. IEEE Computer (December 1996)

[5] Hall, M., Kogge, P., Koller, J., Diniz, P., Chame, J., Draper, J., LaCoss, J., Granacki, J., Brockman, J., Srivastava, A., Athas, W., Freeh, V., Shin, J., Park, J.: Mapping Irregular Applications to DIVA, a PIM-Based Data-Intensive Architecture. In: Proceedings of 1999 Conference on Supercomputing (January 1999)

[6] Judd, D., Yelick, K.: Exploiting On-Chip Memory Bandwidth in the VIRAM Compiler. In: Proceedings of 2nd Workshop on Intelligent Memory Systems, Cambridge, MA (November 12, 2000)

[7] Kang, Y., Huang, W., Yoo, S., Keen, D., Ge, Z., Lam, V., Pattnaik, P., and Torrellas, J.: FlexRAM: Toward an Advanced Intelligent Memory System. In: Proceedings of International Conference on Computer Design (ICCD), Austin, Texas (October 1999)

[8] Landis, D., Roth, L., Hulina, P., Coraor, L., Deno, S.: Evaluation of Computing in Memory Architectures for Digital Image Processing Applications. In: Proceedings of International Conference on Computer Design, pp. 146-151 (1999)

[9] Oskin, M., Chong, F.T., Sherwood, T.: Active Page: A Computation Model for Intelligent Memory. Computer Architecture. In: Proceedings of the 25th Annual International Symposium on Computer Architecture, pp. 192-203 (1998)

[10] Patterson, D., Anderson, T., Cardwell, N., Fromm, R., Keeton, K., Kozyrakis, C., Tomas, R., Yelick, K.: A Case for Intelligent DRAM. IEEE Micro, pp. 33-44 (March/April 1997)

[11] Press, W.H., Teukolsky, S.A., Vetterling, W.T., Flannery, B.P.: Numerical Recipes in Fortran 77. Cambridge University Press, Cambridge (1992) 
[12] Snip, A. K., Elliott, D.G., Margala, M., Durdle, N.G.: Using Computational RAM for Volume Rendering. In: Proceedings of 13th Annual IEEE International Conference on ASIC/SOC, pp. $253-257$ (2000)

[13] Swanson, S., Michelson, K., Schwerin, A., Oskin, M.: WaveScalar. MICRO-36 (December 2003)

[14] Veenstra, J., Fowler, R.: MINT: A Front End for Efficient Simulation of Shared-Memory Multiprocessors. In: Proceedings of MAS-COTS 1994, pp. 201-207 (January 1994)

[15] Wang, K.Y.: Precise Compile-Time Performance Prediction for Superscalar-Based Computers. In: Proceedings of ACM SIGPLAN 1994 Conference on Programming Language Design and Implementation, pp. 73-84 (1994) 